\title{
Anomalous thermomechanical properties and laser-induced densification of vitreous silica
}

\author{
Liping Huang and John Kieffer $^{\mathrm{a})}$ \\ Department of Materials Science and Engineering, University of Michigan, Ann Arbor, \\ Michigan 48109-2136
}

(Received 17 June 2006; accepted 11 August 2006; published online 6 October 2006)

\begin{abstract}
The authors studied the anomalous behaviors of vitreous silica under the combined influence of high temperature and pressure, by using molecular dynamics simulations based on a charge-transfer three-body potential. Accordingly, anomalous properties, such as the minimum in the bulk modulus at $\sim 2-3 \mathrm{GPa}$ and the negative thermal expansion while under pressure, are inherently connected to the ability of the glass to undergo irreversible densification. Their simulations reveal the structural features responsible for this behavior, as well as the extent to which these properties can be tailored through specific processing routes and hence create glass that is less susceptible to radiation damage. (C) 2006 American Institute of Physics. [DOI: 10.1063/1.2357016]
\end{abstract}

Vitreous $\mathrm{SiO}_{2}$ is a leading optical material. For example, lenses made of $\mathrm{SiO}_{2}$ glass enable high-resolution microlithography for the creation of semiconductor integrated circuits using light with 248 and $193 \mathrm{~nm}$ wavelengths. ${ }^{1}$ However, silica glass is known to irreversibly densify under highenergy types of radiation, such as neutrons, ions, electrons, $\mathrm{x}$ rays, and gamma rays, ${ }^{2-5}$ and lately it was found that even longer-wavelength light can cause this effect at sufficiently high intensities. ${ }^{1,6-10}$ Consequently, the refractive index of this material can change under prolonged exposure to highintensity ultraviolet radiation, limiting its usefulness in microlithography. While such behavior is not entirely unexpected, since irreversible densification of silica glass resulting from dynamic or static mechanical compression has been widely observed, ${ }^{11-14}$ the underlying mechanisms are not understood. In particular, it is not known whether the same processes and structural reorganizations are invoked irrespective of the type of external forcing. Given the permanence of the density change, it is natural to assume that some sort of structural transition between distinct noncrystalline structural states takes place, and it is therefore useful in this context to examine the ramifications of the polyamorphism concept. Moreover, since we recently established the role of polyamorphic transitions in several of the anomalous behaviors of silica glass, ${ }^{15-18}$ which include a bulk modulus minimum at $\sim 2-3 \mathrm{GPa},{ }^{19}$ a density maximum at $1800 \mathrm{~K},{ }^{20}$ and a negative thermal expansion (NTE) under pressure, ${ }^{21}$ it is instructive to consider the effects of such anomalies in this analysis as well.

Molecular dynamics (MD) simulations are a powerful tool for studying atomic scale mechanisms underlying materials properties, particularly under conditions that are difficult to observe experimentally, e.g., extremely short time pulses and rapid changes in pressure, temperature, and strain. Several MD studies have been devoted to understanding pressure-induced densification in silica glass. ${ }^{17,22-24}$ Given the limited ability of this technique for directly simulating the interaction between electromagnetic radiation and matter, Kubota et al. $^{25}$ used shock waves to model laser pulses and achieved densification of up to $20 \%$ in fused silica. Wootton et al. $^{26}$ and Zheng et ll. $^{27}$ modeled the laser absorption pro-

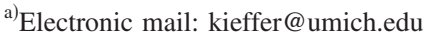

cess in vitreous silica as added kinetic energy in excited $\mathrm{Si}-\mathrm{O}$ bonds, which also produced densification. They proposed that the radiation-induced compaction could be regarded as a localized melting immediately followed by a rapid quench. Because pure vitreous silica exhibits a density maximum at $1800 \mathrm{~K},{ }^{20}$ when the glass is rapidly cooled from this temperature, the higher-density structure (and therefore higher refractive index) is frozen in. They also showed that longer laser pulse duration, higher pressure, and interestingly, higher initial temperature of the glass cause larger densification. This confirms experimental studies by Rothschild et $a l .{ }^{10}$ If the aforementioned "flash melting and quenching" were indeed the only reason for the laser-induced densification, i.e., silica glass essentially remembering the highdensity liquid state it visited, then the initial sample temperature should not matter. Accordingly, the mechanism by which silica densifies upon UV-laser exposure is likely more complex and additional factors may play determining roles. We have investigated this phenomenon using MD simulations and discovered a remarkable coincidence between the propensity of a structure to undergo irreversible densification and the degree to which it exhibits anomalous thermomechanical properties.

Our MD simulations of silica glass were carried out for 3000-particle (1000 Si and $2000 \mathrm{O}$ ) and 8232-particle (2744 $\mathrm{Si}$ and $5488 \mathrm{O}$ ) systems with periodic boundary conditions, using a charge-transfer three-body potential. ${ }^{28}$ No system size effects in excess of statistical errors could be detected. The room temperature silica glasses were obtained by heating and melting cristobalite silica and subsequently quenching the liquid at different cooling rates. Temperature ramping was achieved by velocity scaling and the density adjusted according to the Anderson constant-pressure algorithm. Figure 1 shows that our simulated silica glass has a density maximum at high temperature and that the density of the as-quenched silica glass at room temperature is higher the higher the cooling rate. All these features confirm what has been observed in experiments ${ }^{9}$ and other simulations. ${ }^{20,26,29}$ Accordingly, densification due to freezing in melt densities within the focal volume of the laser exposure would directly depend on the quench rate the exposed glass is subjected to. A higher initial temperature of the glass does not provide for faster quench rates; if anything, the difference between the 


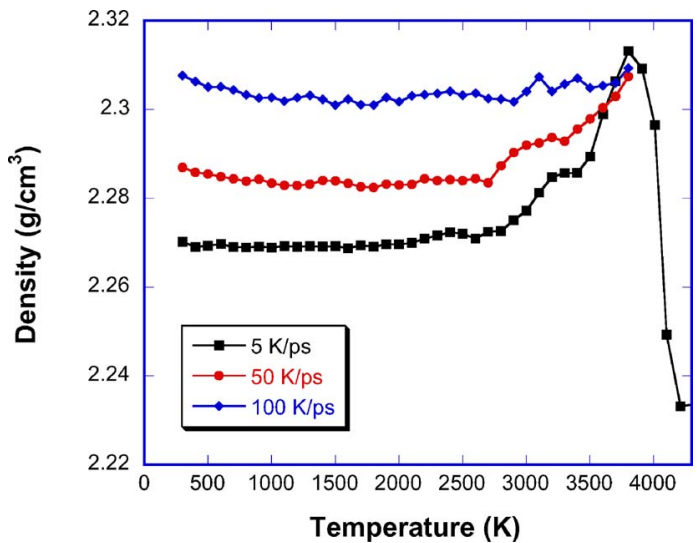

FIG. 1. (Color online) Density evolution with temperature for silica glass quenched with different cooling rates.

temperature corresponding to the density maximum and that of the glass surrounding the focal spot is reduced and sample preheating should therefore have the opposite effect on laserinduced densification than is observed.

Our earlier studies ${ }^{16,17}$ show that the degree of densification that can be achieved in silica glass increases, the higher the temperature at which a given pressure is applied. Similarly, heating silica glass that is subjected to hydrostatic pressure results in the reduction of its volume, i.e., the compressed structure exhibits a behavior akin to NTE, albeit not reversible upon cooling. In fact, the extent of this NTE depends on the magnitude of the applied pressure. Under a hydrostatic tensile stress of $-10 \mathrm{GPa}$, the density of silica glass is essentially independent of temperature; at zero pressure, the glass has a slightly positive thermal expansion; and when surpassing a compressive stress of $\sim 10 \mathrm{GPa}$, thermal expansion is markedly negative and NTE becomes more pronounced with increasing pressure. These observations, which are corroborated by early experimental results, ${ }^{21}$ suggest that pressure induces permanent densification by invoking mechanisms that require thermal activation. Neither temperature nor pressure alone can achieve this effect, i.e., without providing thermal energy there is no permanent densification up to $20 \mathrm{GPa}$ and without applied pressure heating only results in a decrease of density, as expected for a normal solid. These are plausible conditions for densification under laser irradiation: besides the enormous thermal energy imparted by the focused laser light, compressive stresses are generated in the form of shock waves that accompany the laser energy deposition. This provides the combined thermal energy and mechanical work needed to induce the structural rearrangements associated with permanent densification.

Simulations allow for detailed analysis of these structural changes. Upon densification we observe two consecutive processes. One is the abrupt rotation of $\mathrm{Si}-\mathrm{O}-\mathrm{Si}$ bridges resulting in a partial collapse of ring structures without breaking any bonds. The other process is the breaking and re-formation of bonds, transforming smaller rings into larger ones. It is the latter process that leads to permanent densification: larger rings are more flexible; they can fold up on themselves, allowing the structure to pack more efficiently. ${ }^{16,17}$ While under tensile stress, i.e., between $-10 \mathrm{GPa}$ and ambient pressure, the ring size distribution remains unchanged, there is a continuous shift towards larger rings under compressive stress, and this effect becomes more enhanced the higher the temperature.

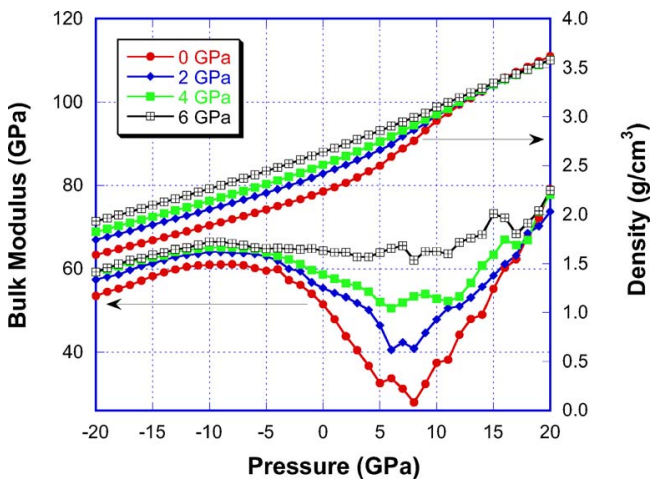

FIG. 2. (Color online) Density and bulk modulus evolution with pressure at $300 \mathrm{~K}$ for silica glass quenched under different pressures.

The first process, i.e., the ring collapse, is actually reversible and in itself does not cause any permanent structural modification. However, the mechanism that facilitates this ring collapse appears to be an essential prerequisite for the permanent densification. As we have shown in pervious simulation studies, this mechanism, i.e., the sudden $\mathrm{Si}-\mathrm{O}-\mathrm{Si}$ rotations analogous to elastic buckling, is also responsible for the material's anomalous behaviors. ${ }^{15-18}$ Evidence that the ring collapse mechanism indeed facilitates the permanent densification of silica glass simply arises from the observation that the anomalous thermomechanical behaviors and the ability to undergo irreversible compaction always disappear simultaneously when subjecting the glass to various conditions described below. In addition to elucidating the mechanism of permanent densification, these conditions delineate potential processing routes for generating glass that is immune to laser irradiation damage.

To illustrate these points, we will describe two simulated experiments. First, under isothermal compression, saturation densities are reached in the released specimens. While these maximum densities are temperature dependent, at any given temperature this maximum cannot be exceeded, even after repeated compression-decompression cycles, i.e., any hydrostatic compaction beyond this point is elastic and reversible. Once this density is achieved, anomalous decrease in bulk modulus with pressure vanishes. ${ }^{11,17}$ Second, we prepared silica glass with different initial structures by quenching the melt from high temperature while applying a compressive hydrostatic stress. The higher this quench pressure, the higher the initial density of silica glass at ambient temperature after releasing the mechanical constraint. Once prepared in this way, the glasses were subjected to hydrostatic compression-decompression cycles at a rate of $0.5 \mathrm{GPa} / \mathrm{ps}$ between -20 and $20 \mathrm{GPa}$ and at $300 \mathrm{~K}$. Figure 2 shows the evolution of the bulk modulus, which was calculated according to $B=\rho(d P / d \rho)$, as a function of the applied hydrostatic stress for four different quench pressures. For quench pressures of $4 \mathrm{GPa}$ or less, the bulk modulus of silica glass decreases with pressure, i.e., behaves anomalously, between -8 and about $6 \mathrm{GPa}$. The bulk modulus minimum at $\sim 6-8 \mathrm{GPa}$ becomes less pronounced with the increase of the quench pressure. Once the quench pressure exceeds $6 \mathrm{GPa}$, the anomalous behavior is absent. Similarly, as shown in Fig. 3(a), a large density increase occurs when compressing a regularly quenched glass to $20 \mathrm{GPa}$ and releasing that constraint, while the glass quenched under $6 \mathrm{GPa}$ is much more resistant to further compression when subjected to the same treatment. Furthermore, NTE under compression disappears 

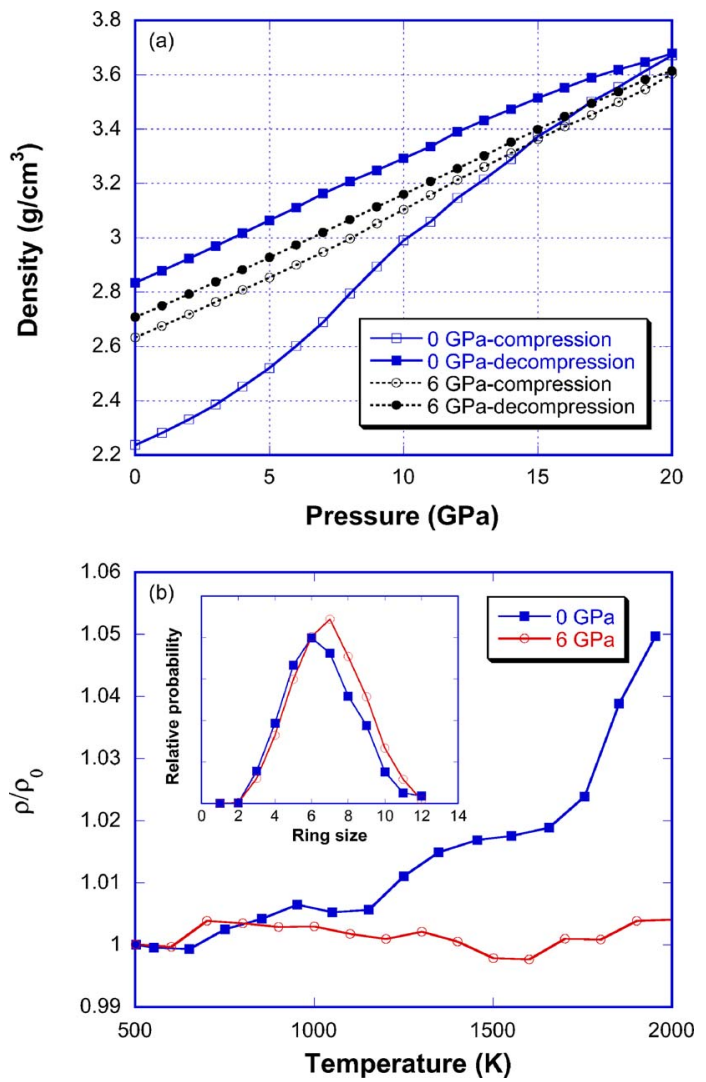

FIG. 3. (Color online) (a) Density evolution during compression and decompression cycle at $1000 \mathrm{~K}$. (b) Relative density change with temperature for silica glass quenched under different pressures. Inset is the ring size distribution of the initial silica glass.

as well [Fig. 3(b)]. When heating glass while a hydrostatic pressure of $10 \mathrm{GPa}$ is applied, NTE is obvious for the regularly quenched system, while for the "pressure-treated" glass the density is temperature-independent within procedural error.

Structural analysis reveals that the ring size distribution gradually shifts towards larger rings when glasses are produced under higher pressures [see inset of Fig. 3(b)]. In contrast to large rings, which pack better, smaller rings that have constrained convex geometries create more free volume in the structure. Evidently, it is this free volume that allows for the implosive collapse of rings described above as underlying anomalous behaviors of the glass. Hence, when processed under conditions that favor formation of larger rings, e.g., high pressure, both the negative dependence of the elastic modulus on pressure and NTE vanish. At the same time, the capacity for further densification is eliminated, which raises the question whether there could be a causal relationship between these thermomechanical anomalies and densification.

We submit that there is. The anomalous behavior shown in Fig. 2 is reflective of an inherently unstable structural state. The softening of silica glass upon compression means progressively less resistance to further deformation. In this sense, densification is a self-catalyzing process that invites additional compaction and inevitably leads to a catastrophic condition at the molecular level, i.e., the collision of atoms and re-formation of the local bonding structure. Accordingly, irradiation damage is based on three concurrent phenomena: (i) the incident laser pulse creates the mechanical actuation that compacts the structure, i.e., through the accompanying shock waves, (ii) the resulting deformation invokes subtle structural transitions that make the material more susceptible to further densification, and (iii) laser heating provides the thermal activation for the necessary structural rearrangements. The density maximum in the liquid is merely a manifestation of the same innate feature that triggers the structural collapse but does not actually represent the path to densification.

In this process, the inherent instability of network rings in regularly quenched glass, as evidenced by a progressive weakening of the structure upon initial compaction, is key to facilitating permanent densification. By preparing silica glass in ways that eliminate anomalous thermomechanical behaviors, e.g., by quenching a melt under pressure or by performing a chemical modification of the network with a similar effect, the propensity of the glass to undergo irreversible densification can be eradicated. Such pressure-treated silica glass can potentially increase the lifetime of many optical components.

This work was supported by the National Institute of Standards and Technology and the National Science Foundation.

${ }^{1}$ T. P. Seward, C. Smith, N. F. Borrelli, and D. C. Allan, J. Non-Cryst. Solids 222, 407 (1997).

${ }^{2}$ E. P. EerNisse, J. Appl. Phys. 45, 167 (1974).

${ }^{3}$ C. B. Norris and E. P. EerNisse, J. Appl. Phys. 45, 3876 (1974).

${ }^{4}$ T. A. Dellin, D. A. Tichenor, and E. H. Barsis, J. Appl. Phys. 48, 1131 (1977).

${ }^{5}$ W. Primak, J. Appl. Phys. 49, 2572 (1977).

${ }^{6}$ K. Awazu, J. Non-Cryst. Solids 337, 241 (2004).

${ }^{7}$ D. C. Allan, C. Smith, N. F. Borrelli, and T. P. Seward, Opt. Lett. 21, 1960 (1996).

${ }^{8}$ N. F. Borrelli, C. Smith, D. C. Allan, and T. P. Seward, J. Opt. Soc. Am. B 14, 1606 (1997)

${ }^{9}$ C. Fiori and R. A. B. Devine, Appl. Phys. Lett. 47, 361 (1985).

${ }^{10}$ M. Rothschild, D. J. Ehrlich, and D. C. Shaver, Appl. Phys. Lett. 55, 1276 (1989).

${ }^{11}$ M. Grimsditch, Phys. Rev. Lett. 52, 2379 (1984).

${ }^{12}$ R. J. Hemley, H. K. Mao, P. M. Bell, and B. O. Mysen, Phys. Rev. Lett. 57, 747 (1986).

${ }^{13}$ C. Meade, R. J. Hemley, and H. K. Mao, Phys. Rev. Lett. 69, 1387 (1992).

${ }^{14}$ G. D. Mukherjee, S. N. Vaidya, and V. Sugandhi, Phys. Rev. Lett. 87, 195501 (2001).

${ }^{15}$ L. P. Huang, L. Duffrene, and J. Kieffer, J. Non-Cryst. Solids 349, 1 (2004).

${ }^{16}$ L. P. Huang and J. Kieffer, Phys. Rev. B 69, 224203 (2004).

${ }^{17}$ L. P. Huang and J. Kieffer, Phys. Rev. B 69, 224204 (2004).

${ }^{18}$ L. P. Huang and J. Kieffer, Glass Sci. Technol. (Offenbach, Ger.) 77, 124 (2004).

${ }^{19}$ C. Meade and R. Jeanloz, Phys. Rev. B 35, 236 (1987).

${ }^{20}$ J. E. Shelby, Introduction to Glass Science and Technology, 2nd ed. (The Royal Society of Chemistry, Cambridge, 1997), p. 138.

${ }^{21}$ R. Roy and H. M. Cohen, Nature (London) 190, 798 (1961).

${ }^{22}$ W. Jin, R. K. Kalia, and P. Vashishta, Phys. Rev. B 50, 118 (1994).

${ }^{23}$ J. S. Tse, D. D. Klug, and Y. Le Page, Phys. Rev. B 46, 5933 (1992).

${ }^{24}$ K. Trachenko and M. T. Dove, J. Phys.: Condens. Matter 14, 7449 (2002).

${ }^{25}$ A. Kubota, M. J. Caturla, J. S. Stolken, and M. D. Feit, Opt. Express 8, 611 (2001).

${ }^{26}$ A. Wootton, B. Thomas, and P. Harrowell, J. Chem. Phys. 115, 3336 (2001).

${ }^{27}$ L. Q. Zheng, J. C. Lambropoulos, and A. W. Schmid, J. Non-Cryst. Solids 351, 3271 (2005).

${ }^{28}$ L. P. Huang and J. Kieffer, J. Chem. Phys. 118, 1487 (2003).

${ }^{29}$ K. Vollmayr, W. Kob, and K. Binder, Phys. Rev. B 54, 15808 (1996). 